\title{
New data on the competition between definites and indefinites
}

Nadine Bade \& Florian Schwarz*

\begin{abstract}
In this paper, we report on four experiments investigating obligatory presupposition effects. Specifically, we look at the inferences arising from not using presupposition triggers when their use is supported by the context. We compare these inferences and the contextual factors for their derivation to presuppositions and implicatures. Extending previous work, we explore not only the English definite determiner "the" but also the dual "both" and their respective competition with the universal quantifiers "every" and "all".
\end{abstract}

Keywords. presuppositions, non-presuppositions, implicatures

1. Introduction. In this paper, we present a set of novel experimental data from English shedding light on the non-uniqueness effect associated with the indefinite determiner, as illustrated by the classic example in (1).

(1) \#A father of the victim arrived at the crime scene.

(Heim 1991)

$\rightsquigarrow$ there is more than one father of the victim

The oddness of (1) is usually attributed to the fact that indefinite determiner phrases (DP) cannot refer to unique objects due to a blocking effect evoked by the definite DP "the father of the victim". The observation that definite marking must be used when it can be - given the presupposition of uniqueness is fulfilled - has been accounted for by postulating a general principle Maximize Presupposition, which has received substantial attention in the recent literature (Heim 1991, Chemla 2008, Percus 2006, Sauerland 2008, Singh 2011, Schlenker 2012, Marty 2017, Anvari 2018, Spector \& Sudo 2017, Rouillard \& Schwarz 2017, Marty \& Romoli 2020).

In this paper, we look at inferences arising as a result of reasoning with Maximize Presupposition and compare them to presuppositions and implicatures in different experimental settings.

To test the generality of the phenomenon exemplified by (1) we also look at the competition between quantifiers "all" and "every" with the presuppositionally stronger "both" and "the", respectively, see (2-a) and (2-b).

(2) a. John broke $\{\#$ all / both $\}$ of his arms.

b. $\quad\{\#$ Every / The $\}$ sun is shining.

\section{Background.}

2.1. Theoretical Background. The principle Maximize Presupposition (Heim 1991) has been posited as a general pragmatic principle to account for the obligatory insertion of presupposition triggers:

Maximize Presupposition (Heim 1991) Make your contribution presuppose as much as possible!

*Nadine Bade, University of Potsdam (nadine.bade@uni-potsdam.de) \& Florian Schwarz, University of Pennsylvania (florians@ling.upenn.edu). 
It explains why (3-b) is preferred over (3-a), given world knowledge that everyone has a unique (biological) father. (Parallel explanations extend to the other examples.)

(3) a. \#A father of the victim arrived at the crime scene.

b. The father of the victim arrived at the crime scene.

Heim (1991) argued that the maxim of quantity could not account for these cases under the theoretical assumption that (3-a) and (3-b) are contextually equivalent (=equally informative assuming the truth of the presupposition) but differ in the definedness conditions they introduce. Postulating a separate principle for these phenomena also aligns with data suggesting that inferences evoked by presuppositional competition show behavior which is different from presuppositions and implicatures (Sauerland 2008). Specifically, these inferences (called presuppositional implicatures in the following) have been argued to have a weaker status (resist strengthening), and project at the same time. That is, the oddness effect of the presuppositionally weaker alternative is preserved under holes for presuppositions, such as negation, see (4).

(4) a. \#Not all arms of John are broken.

b. \#I did not see a father of the victim.

However, this line of argument has been challenged in the more recent literature. Most of the challenging data discussed revolve around claims that strengthening of presuppositional implicatures is possible, and that their derivation is mandatory under certain circumstances (Chemla 2008, Magri 2009, Marty 2017, Elliott \& Sauerland 2019). (3-a) is a prominent example of this effect. The oddness of the sentence seems to result from the non-uniqueness inference being mandatory, and blind to common knowledge (Singh 2011, Magri 2009). Based on these challenging data, presuppositional implicatures have been claimed to be derived by the same mechanism as implicatures.

Nonetheless, the theoretical status of these inferences still remains unclear. There is no agreement on whether implicatures and presuppositional implicatures should be treated completely on a par (see e.g. Rouillard \& Schwarz (2017) for discussion). It has been claimed that, just like for implicatures, the presence and relevance of the alternative, the knowledge state of hearer and speaker, as well as ease of accommodation are crucial factors impacting whether presuppositional implicatures arise and are strengthened (Chemla 2008, Marty 2017, Elliott \& Sauerland 2019). The goal of the experiments reported below was to test the predictions of different theories with regard to what role these factors play.

2.2. Previous experimental WORK. Several claims in the theoretical literature about the role of alternatives for implicatures have been supported by experimental results. Both the presence and relevance of the alternative play a role in deriving implicatures (Bott \& Chemla 2016, Rees \& Bott 2018, Degen \& Tanenhaus 2015). They have been argued to be one deciding factor for whether the processing of implicatures is delayed (Huang \& Snedeker 2009, 2011), or immediate (Grodner et al. 2010). Furthermore, implicatures have not only been shown to differ from literal meaning in processing but also to differ from presuppositions (Bill et al. 2018).

There are fewer experimental investigations of the processing of presuppositional implicatures. Most of the existing literature focuses on the difference between indefinite and definite determiners. Kirsten et al. (2014) find in an EEG experiment that unmet non-uniqueness infer- 
ences evoke higher processing costs for the indefinite than unmet uniqueness presuppositions for the definite determiner. They attribute this difference to additional cognitive load demanded by the introduction of a new discourse referent with the indefinite.

Another set of reading time studies tested the use of definites versus indefinites referring to stereo-typically unique items in contexts in which they are typically unique (e.g. stove in a kitchen) or not (e.g. stove in an appliance store) Clifton Jr (2013). Clifton found that interactions between contexts and determiner in reading times only emerged if the experiment involved a secondary arithmetic task, which is argued to lead to deeper processing resulting in participants forming a more complete situation model.

Mouse-tracking data from Schneider et al. (2019) support a view where unmet uniqueness for the definite and unmet non-uniqueness for the indefinite do not evoke parallel processing behavior. These data also stress the relevance of the presuppositional alternative for the indefinite.

Further evidence for differences between definite and indefinite comes from an eye-tracking study reported in Bade \& Schwarz (2019a). They suggest that, if the inference is drawn, nonuniqueness evokes different gaze patterns than those arising when deriving a uniqueness presupposition. Specifically, in line with Schneider et al. (2019)'s results, the indefinite seems to involve more consideration of the alternative.

In contrast, an eye-tracking experiment reported in Bade \& Schwarz (2019b) reveals that if the contextual conditions for drawing the non-uniqueness are fully met the processing differences between indefinites and definites disappear. The results suggest that these contextual conditions involve complete awareness of the definite alternative and its relevance. In the experiment, this was achieved by including a production task providing definite and indefinite as alternatives. A similar pattern is observed in a mouse-tracking study by (Schneider et al. 2020). In the study, participants were also presented with a production task first. The results show that both determiners initiate immediate movements towards the target, with barely any differences between determiners for the relevant measures considered. Again, this suggest that awareness of the alternative matters, in that it makes the patterns for the determiners indistinguishable.

3. Experiments. We ran four experiments in total. A first goal was to establish contexts where inferences involving reasoning over presuppositional alternatives reliably arise. The second goal was to identify the factors that make these alternatives salient, and compare them to the ones of other scale types.

\subsection{STUdy OnE: PiCTURE SELECTION.}

3.1.1. Aims. The aim of this study was to test whether presenting material as a dialog affects target choices of the two determiners differently. A weakness of previous studies was that they left the overall discourse situation implicit or underspecified. In the current experiment, we made it clear that speaker and hearer had knowledge of the situation, including the number of items talked about, and that thas number was relevant.

3.1.2. Design And MATERial. We used a simple $2 \times 2$ design with DETERMiner and PiCtURE COMPETITION fully crossed. Sentences with an indefinite or definite determiner such as given in (5) were used in a comic strip, see Figure 1a and 2a.

$\{\mathrm{A} / \mathrm{The}\}$ shirt in my closet has a stain on it.

Nadine Bade and Florian Schwarz:

New data on the competition between definites and indefinites. 
There were two different pairs of pictures shown as competing options along this comic, see Figures 1 and 2. The first competition was between a picture with a single stained shirt and a picture with three stained shirts. The second competition was between a single stained shirt picture and a picture with three shirts, one of which was stained. There were, moreover, filler items using similar picture types but containing sentences with "only" or plurals with negation in the comic strip (the same fillers were used for all experiments).

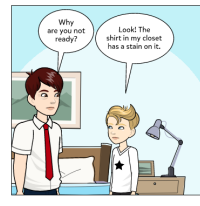

(a) Sample comic with "the"

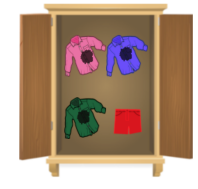

(b) 3 shirts - 3 stains

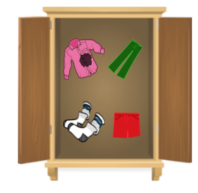

(c) 1 shirt - 1 stain

Figure 1: 3shirts-1stain versus 3shirts-1stain

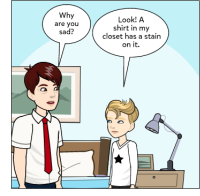

(a) Sample comic with "a"

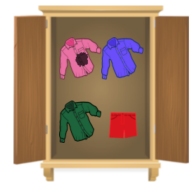

(b) 3 shirts - 1 stain

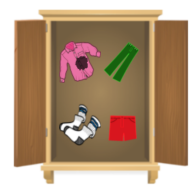

(c) 1 shirt - 1 stain

Figure 2: 3shirts-3stains versus 3shirts-1stain

Participants' task was to pick the picture they thought matched the comic strip they saw.

3.1.3. Predictions. Sentences with an indefinite such as "A shirt in my closet has a stain on it" should come with an inference of non-uniqueness ('there is not exactly one shirt in the closet'). Accordingly, we predicted that pictures with non-unique objects would be targets for indefinites. Conversely, sentences with the definite should come with a presupposition of uniqueness ('there is exactly one shirt in the closet'). As a result, pictures with unique objects are predicted to be targets for definites. However, a further complication, attested in previous results, arises from an implicature concerning the number of items of which the predicate is true ('there is exactly one shirt with a stain on it'), which seems to introduce a certain amount of infelicity relative to pictures where it is not met. We therefore expect an interaction of PICTURES and DETERMINER: Whereas the definite determiner should exhibit high rates of choices of unique object-pictures in both conditions, which align both with its presupposition and this implicature, the presuppositional implicature introduced by the indefinite determiner competes with this implicature in the picture pairing in Figure 1; but in the condition illustrated in Figure 2, where both pictures satisfy the 'exactly-one'-implicature, participants should choose the 3 shirts-1stain picture type as it is the only choice that matches the presuppositional implicature.

3.1.4. RESULTS. We used a generalized linear mixed effects model analysis to compare the rate of 1shirt-1stain type picture choices. The model had random slopes for participants and random intercepts for items. We find a significant interaction between picture type and determiner (see stats in Table 1). In line with predictions, the choice for the unique object picture is at ceiling for the definite across competitors. For the indefinite, a clear choice for the 3 shirt picture - in line 
with the presuppositional implicature - only transpired when the implicature was met (with just one stained shirt), otherwise, the 1shirt-1stain picture was the predominant choice, presumably to avoid incompatibility with the one stain implicature.

\begin{tabular}{lllll} 
& Estimate & Std.Err & $\mathrm{z}$ value & $\mathrm{p}$ value \\
\hline 3shirts-3stains vs 3shirts - 1stain:the & -3.5605 & 0.4067 & -8.754 & $<2 \mathrm{e}-16 * * *$
\end{tabular}

Table 1: Output of glmer for interaction between picture context and determiner

To see whether determiners differed in the rate of target choices for the picture condition where both pictures satisfied the implicature, we looked at the interaction between context and determiner with target coded as the dependent variable. We see no difference in target choices between determiners when the implicature was satisfied by both pictures $(\hat{\beta}=-1.83, \mathrm{SE}=1.112, \mathrm{z}$ $=-1.645, p=0.3532)$. Significance was calculated based on least square means using the emmeans package in R. The statistical results are summarized in Figure 3.

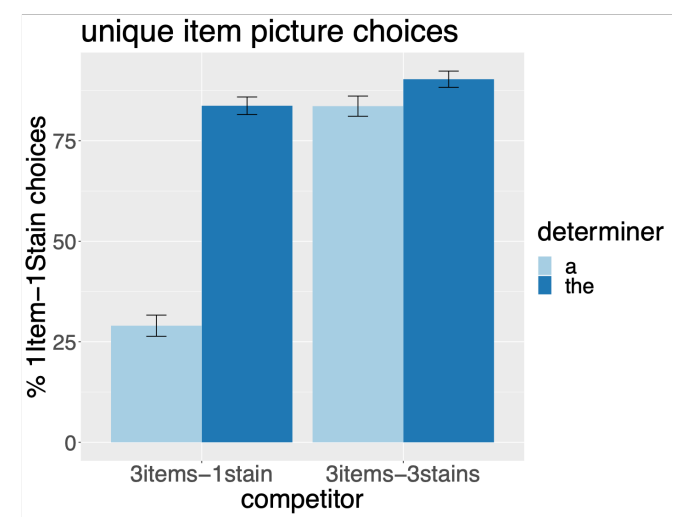

Figure 3: Experiment 1 - Percentage of choices for 1shirt-1stain type picture by determiner and competitor picture

In sum, we find evidence of both the presupposition of the definite and the presuppositional implicature of the indefinite impacting picture choice rates. The rate of target choices for the indefinite and definite is the same when no interfering implicature is at play. Once it is, the impact of the presuppositional implicature becomes minimal, as a desire to avoid an incompatibility with the one-stain implicature seems to dominate choice patterns.

\subsection{Study two: Acceptability Rating.}

3.2.1. Aims. The aim of the second study was to directly evaluate the acceptability of the sentences in the various picture contexts provided to speakers to see whether any oddness arises, and to which degree, for violating the different inference types under scrutiny.

3.2.2. DESIGN. The design is $2 \times 2$ with the factors DETERMINER and PICTURE TYPE fully crossed. The latter was a group factor, i.e. people overall saw only one picture type. We tested individual picture types - 1shirt-1stain, 3shirts-1stain, 3shirts-3stains - with one of the two determiners. We asked people how natural they find the sentence in the given picture context on a scale from 1-7 (completely natural). 
Predictions The acceptability of the 1shirt-1stain picture should be higher than the 3 shirts-1stain picture for the definite, due to the latter violating the uniqueness presupposition, or alternatively requiring domain restriction to make the presupposition true. Reversely, for the indefinite, the 3 shirts-1stain picture should be more acceptable than the 1shirt-1stain picture, in light of its presuppositional implicature of non-uniqueness. Given theoretical assumptions the 1shirt-1stain picture should furthermore be more acceptable with the definite than indefinite. We thus predicted an interaction.

3.2.3. RESUlts. Assuming an ordinal scale, we used a cumulative link model analysis and the clmm function in $\mathrm{R}$ to analyze the acceptability rating data. We find an interaction between picture type and determiner, see Figure 4 and stats in table 2, but only for the comparison between 1shirt1 stain and 3shirts-1stain. However, numerical differences are very small.

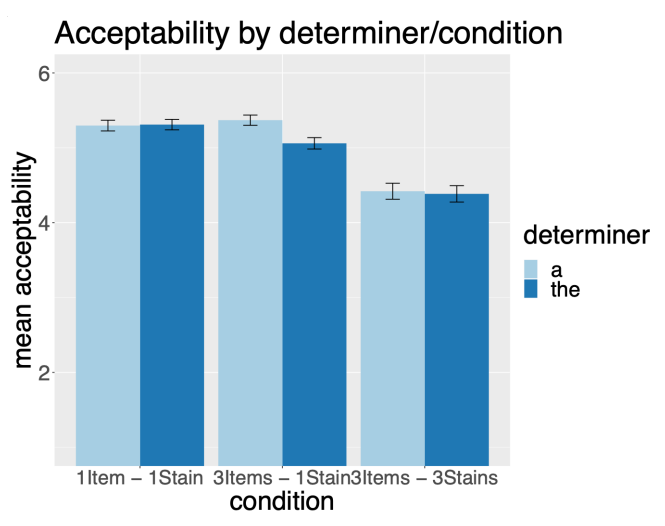

Figure 4: Experiment 2 - Average acceptability by determiner and picture type

\begin{tabular}{lllll} 
& Estimate & Std.Err & $\mathrm{z}$ value & $\mathrm{p}$ value \\
\hline 3shirts - 1stain:the & -1.145046 & 0.432405 & -2.648 & $0.00809 * *$
\end{tabular}

Table 2: Output of clmm for interaction between determiner and picture

Looking at contrasts, we see a difference between determiners only for the 3 shirts-1stain picture, see Table 3.

\begin{tabular}{lllll} 
contrast & Estimate & Std.Err & z.ratio & p value \\
\hline 3shirts - 1stain a vs the & 1.3027 & 0.335 & 3.893 & $0.0014^{* *}$
\end{tabular}

Table 3: Output of EMMEANs for pairwise contrasts between determiner for 3items - 1 stain picture type

In sum, we see no substantial differences between determiners, suggesting that in the contexts given, their use is equally acceptable. This contrasts with the picture selection data reported above, which suggest awareness of the inferences these determiners come with. ${ }^{1}$

\subsection{STUdY THREE: COVERED BOX.}

\footnotetext{
${ }^{1}$ The results are not likely to be the result of a ceiling effect, as we observe rather low ratings for our complex filler items.
} 
3.3.1. AIM. The goal of the third experiment was to see whether a covered box paradigm would change response patterns. In addition to changing the task for experiment three, we added a contextual manipulation to test whether increasing the relevance of uniqueness being true would boost choices based on presuppositional implicatures of the indefinite.

3.3.2. Design And material. Again, we use a $2 \times 2$ design with COnteXt and Determiner being fully crossed. CONTEXT was a group factor. In the first context, the question posed by the parent in the picture was more neutral, see (6-a). In the second context, the question was more specific to the situation, see (6-b).

(6) a. Context "sad": Why are you sad?

b. Context "why": Why are you not getting dressed?

c. Target: $\{\mathrm{A} / \mathrm{The}\}$ shirt in my closet has a stain.

Both contexts were paired with an overt picture showing a single shirt, which is stained, and a picture which is partially covered by boxes and revealed nothing about shirts, see Figure 5 .

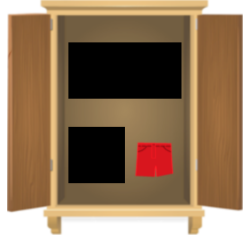

(a) Example of a picture with a covered box

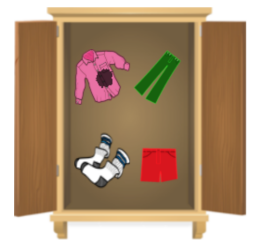

(b) 1 shirt - 1 stain

Figure 5: Picture condition for both contexts

Participants were instructed to choose the covered box picture if they thought the overt picture did not match the context.

3.3.3. Predictions. The prediction was that the indefinite is affected more by the context manipulation than the definite, i.e. that there are more overt target choices with the "sad" context than the "why" context for"a shirt"-sentences, but an equal amount of target choices for sentences containing "the". The result should be an interaction between CONTEXT and DETERMINER.

3.3.4. RESULTS. We analyzed the rate of overt picture choices using a generalized linear mixed effect model analysis. We find neither an effect of context, nor an effect of determiner for either of the context types.

Overall, we see no choices of the covered box picture based on the non-uniqueness inference of the indefinite, see Figure 6. The rate of choices for the overt picture depicting a unique stained item, here a shirt, were at ceiling for both determiners and across contexts.

\subsection{Study 4: Extension of The Design to ALl/Both.}

3.4.1. Aims. The aims of experiment 4 were two-fold. First, the goal was to extend the data on competition of the definite with indefinite to the competition between the dual "both" and universal quantifier "all", as well as the competition of the definite with the universal quantifier "every". Second, the goal was to compare the non-duality and non-uniqueness presuppositional implicatures of universal quantifiers to indirect implicatures evoked by "not all"/“not every" (="some"). 


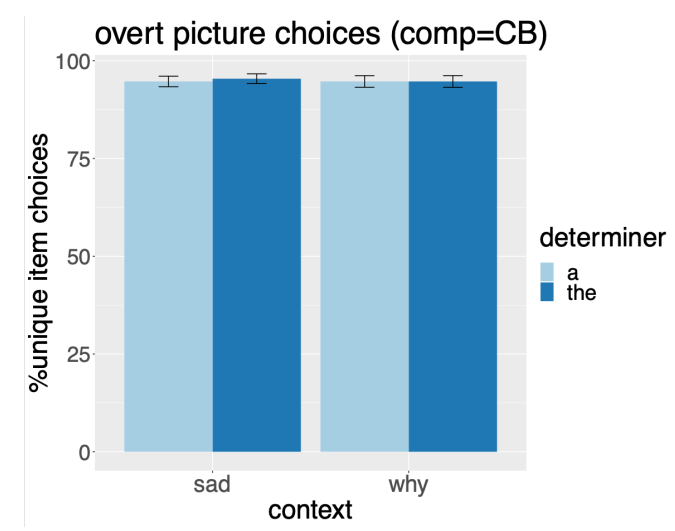

Figure 6: Experiment 3 - Rate of overt picture choices by context and determiner

3.4.2. Design AND material. Sentences used in the comic strip for experiment 4 contained "both" and "all" with and without negation, see (7-a) and (7-c). Furthermore, we tested the competition between "every" and "the" with sentences such as (7-b). Participants saw both competitors for each pair, but only one of the pairs. Negation was also a group factor, i.e. participants saw "both" and "all" either always with or always without negation.

(7) a. The shirts in my closet $\{$ all/both $\}$ have a stain on them.

b. $\quad\{$ Every/ The $\}$ shirt in my closet has a stain on it.

c. The shirts in my closet are not $\{$ all/both $\}$ stain-free.

The positive sentences in (7-a) and (7-b) were only paired with one picture competition each. For the competition between dual and "all", one picture made the presuppositional implicature of non-duality true, see Figure 7b, the other made the presupposition of duality true, see Figure 7a.

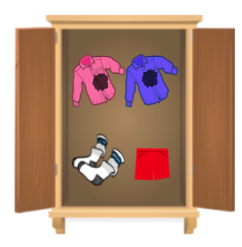

(a) 2 shirts - 2 stains

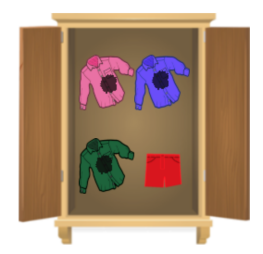

(b) 3 shirts - 3 stains

Figure 7: Picture competition for "both" and "all"

For the competition between definite and "every", there was a picture in which 3 of 3 shirts were stained (making non-uniqueness true), and a picture where there was a single stained shirt (making uniqueness true), see Figures $8 \mathrm{a}$ and $8 \mathrm{~b}$.

For the experiment with negation, there are three individual pictures types, see 9.

Negated universal quantifiers have an indirect "some"-implicature as well as an non-duality presuppositional implicature (projected). "Both" and "not both" sentences have a presupposition of duality. Given these assumptions, the properties of pictures depicted in 9 with regard to the inferences associated with "not all" and "not both" are given in Table 4 below.

There were two relevant picture competitions, one that paired the 2 shirts-1stain with the 


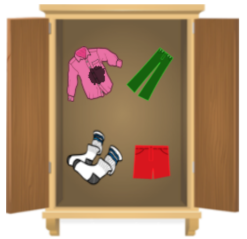

(a) 1 shirt - 1 stain

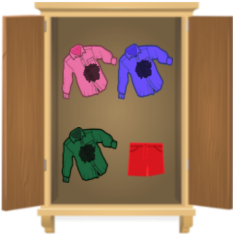

(b) 3 shirts - 3 stains

Figure 8: Picture competition for "every" and "the"

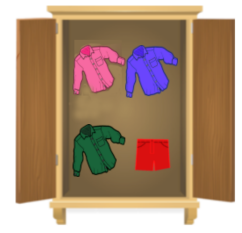

(a) 3 shirts - 0 stains

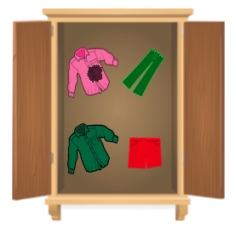

(b) 2 shirts - 1 stain

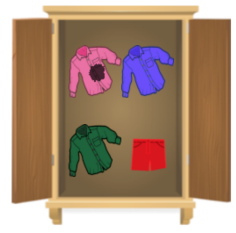

(c) 3 shirts - 1 stain

Figure 9: Picture types paired for "not all" and "not both" sentences

\begin{tabular}{llll} 
& 2SHIRTS-1STAIN & 3SHIRTS - 1STAIN & 3SHIRTS - 0STAINS \\
\hline 1) not both (impl./pres.) & TRUE/TRUE & TRUE/FALSE & FALSE/FALSE \\
2) not all (impl./pres. impl.) & TRUE/FALSE & TRUE/TRUE & FALSE/TRUE
\end{tabular}

Table 4: Status of inferences associated with "not all" and "not both" for each picture type

3 shirts-1stain picture. This picture competition allowed us to compare violated presuppositional implicature with violated presupposition. The indirect implicature is true in both cases. The second pairing is a 2 shirts-1stain versus 3 shirts-0stain picture pairing. It compares violated presuppositional implicatures of non-duality (former) with violated indirect implicature (latter). The two pairings appeared with both type of determiners.

3.4.3. Predictions. We predicted choices to be driven by the presuppositional implicatures of non-duality and non-uniqueness associated with universal quantifiers. For both, participants should choose the target with three of three stained shirts. The definite's presupposition should drive people's choice to the picture with a unique stained shirt. The presupposition of "both" is predicted to make people choose the picture with exactly two shirts, both stained. Regarding negation, we predicted a similar pattern. The projecting presuppositions and presuppositional implicatures should make the 3shirts-1stain picture the target for "not all", and the 2shirts-1stain picture the target for "not both", respectively. If these scales work similarly to the one containing $\{\mathrm{a}$, the , we predict there to be more choices of the non-duality violating picture for "not both" (2shirts-1stain) given that the competitor (3shirts-0stain) violates the indirect "some"-implicature.

3.4.4. RESUlTS. The findings for the cases without negation are exactly as predicted. For both "all" and "every" we see choices based on their presuppositional implicatures, both significantly differ for the choices for the definite and "both", see Table 5.

The results look much more reliable than for indefinites, with target choices being at ceiling, 


\begin{tabular}{lllll} 
& Estimate & Std.Err & $\mathrm{z}$ value & $\mathrm{p}$ value \\
\hline det both & -5.6115 & 0.8085 & -6.941 & $3.90 \mathrm{e}-12 * * *$ \\
det the & 21.207454 & 0.001823 & 11636 & $<2 \mathrm{e}-16 * * *$
\end{tabular}

Table 5: Output of glmer for main effect of determiner, in the case of "both" compared to "all", for "the" compared to "every"

see Figures 10a and 10b. For the cases involving negation, we see the predicted interaction, see Table 6.

\begin{tabular}{lllll} 
& Estimate & Std.Err & $\mathrm{z}$ value & $\mathrm{p}$ value \\
\hline 2shirts - 1stain vs. 3shirts-0stains:notboth & 7.9661 & 1.0573 & 7.534 & $4.91 \mathrm{e}-14 * * *$
\end{tabular}

Table 6: Output for glmer for interaction between determiner and picture pairing

This interaction is driven by the fact that when the implicature is true, choices for the universal quantifier are driven by non-duality, and choices for "both" driven by duality. However, in the pairing where one picture is implicature violating, participants choose the 2shirts-1stain picture with both "not all" and "not both", irrespective of the non-duality presuppositional implicatures being violated, see Figure 10c.
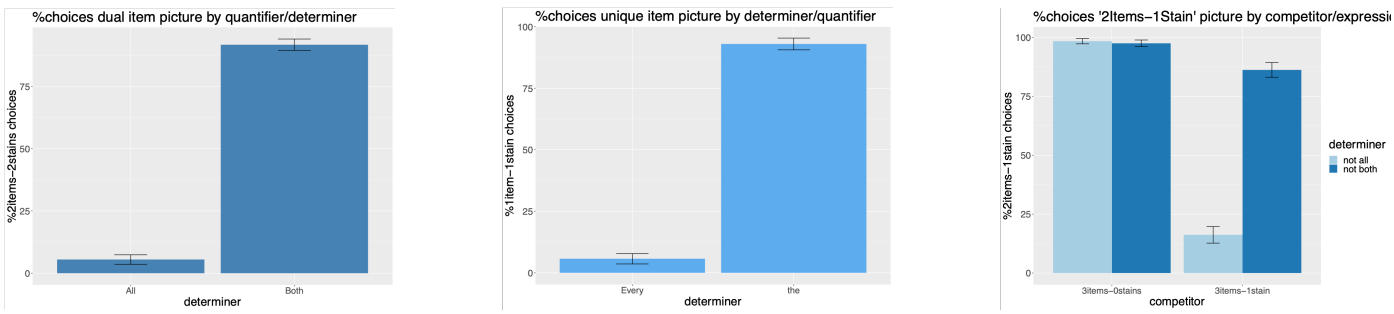

(a) Percentage of choices for (b) Percentage of choices for (c) Percentage of 2 shirts - 1 picture with exactly one shirt by picture with exactly two shirts stain picture by determiner and determiner

by determiner competitor picture

Figure 10: Results experiment 4

4. Discussion. To sum up, non-uniqueness inferences associated with indefinite determiners drive picture choices (Exp 1), but only when its 'exactly-one' implicature is true (Exp 1). This contrasts with previous findings where choices were not relying on non-uniqueness inferences to the same degree (Bade \& Schwarz 2019a). The difference between the studies lay predominantly in the discourse situation being much more specified in the current experiments. We take this to suggest that the knowledge state of speaker and hearer as well as relevance of the alternative play a crucial role in deriving presuppositional implicatures. Our results thus further stress the importance of making the alternative with the definite competitor salient.

However, we see no effect of non-uniqueness in acceptability (Exp 2) or when the competitor picture is a covered box, even with additional contextual pressure (Exp 3). The findings suggest that both determiners are felicitous choices both in presupposition and presuppositional implicature violating contexts. These findings stand in contrast with previous observations that both violations give rise to pragmatic oddness (e.g. in the cases of "A father of the victim..." or "A 
highest mountain...”). They suggest that the definite alternative is not automatically activated (e.g. by the lexicon), even in a context that makes it salient, but must be raised by an overt picture. Our findings also raise a methodological issue: in how far did participants perceive the inferences to be contextually entailed with pictures?

For the competition between "every" vs. "the" and "both" vs. "every" (Exp 4) we see a clear effect of non-uniqueness and non-duality inferences with both universal quantifiers in picture choices. This contrasts with the less reliable findings for the indefinite. It suggests that the ambiguity of the indefinite may play a role, and different usages may associated with different sets of competitors, depending on what is at issue. A parallel we observe is that, as for indefinites, the choice falls on the presuppositional implicature violating picture (only) if the competitor is implicature violating. Why one would take precedence over the other is an open question for any theory, but especially under those theories that propose unique operator analyses where implicatures and presuppositional implicatures are derived by the same mechanism (Marty 2017). If this approach is adopted more needs to be said about the relevance of the presuppositional alternative, when it must be activated or can be ignored. Further research is needed complementing the methodology used here to understand this relation between focus, competition and alternatives in the domain of presupposition versus assertion better.

\section{References}

Anvari, Amir. 2018. Logical integrity. In Proceedings of Semantics and Linguistic Theory 28, vol. 28, 711. Linguistic Society of America. 10.3765/salt.v28i0.4419.

Bade, Nadine \& Florian Schwarz. 2019a. An experimental investigation of antipresuppositions. In Ava Creemers \& Caitlin Richter (eds.), Proceedings of Penn Linguistics Colloquium 42, $31-40$.

Bade, Nadine \& Florian Schwarz. 2019b. (In-)definites, (Anti-)uniqueness, and uniqueness expectations. In Proceedings of CogSci 2019, 119-125.

Bill, Cory, Jacopo Romoli \& Florian Schwarz. 2018. Processing presuppositions and implicatures: Similarities and differences. Frontiers in Communication 3. 44.

Bott, Lewis \& Emmanuel Chemla. 2016. Shared and distinct mechanisms in deriving linguistic enrichment. Journal of Memory and Language 91. 117-140.

Chemla, Emmanuel. 2008. An epistemic step for antipresuppositions. Journal of Semantics 25(2). 141-173.

Clifton Jr, Charles. 2013. Situational context affects definiteness preferences: Accommodation of presuppositions. Journal of Experimental Psychology: Learning, Memory, and Cognition 39(2). 487.

Degen, Judith \& Michael K Tanenhaus. 2015. Processing scalar implicature: A constraint-based approach. Cognitive science 39(4). 667-710.

Elliott, Patrick \& Uli Sauerland. 2019. Ineffability and Unexhaustification. In M.Teresa Espinal, Elena Castroviejo, Manuel Leonetti, Louise McNally \& Cristina Real-Puigdollers (eds.), Proceedings of Sinn und Bedeutung 23, 399-412.

Grodner, Daniel J., Natalie M. Klein, Kathleen M. Carbary \& Michael K. Tanenhaus. 2010. "some," and possibly all, scalar inferences are not delayed: Evidence for immediate prag- 
matic enrichment. Cognition 116(1). 42 - 55.

Heim, Irene. 1991. Artikel und Definitheit. In Arnim von Stechow \& Dieter Wunderlich (eds.), Semantics: An international handbook of contemporary research, 487-535. Berlin: Mouton de Gruyter.

Huang, Yi Ting \& Jesse Snedeker. 2009. Online interpretation of scalar quantifiers: Insight into the semantics-pragmatics interface. Cognitive Psychology 58(3). 376 - 415.

Huang, Yi Ting \& Jesse Snedeker. 2011. Logic and conversation revisited: Evidence for a division between semantic and pragmatic content in real-time language comprehension. Language and Cognitive Processes 26(8). 1161-1172.

Kirsten, Mareike, Sonja Tiemann, Verena C. Seibold, Ingo Hertrich, Sigrid Beck \& Bettina Rolke. 2014. When the polar bear encounters many polar bears: event-related potential context effects evoked by uniqueness failure. Language, Cognition and Neuroscience 29(9). 1147-1162.

Magri, Giorgio. 2009. A theory of individual-level predicates based on blind mandatory scalar implicatures. Natural Language Semantics 17(3). 245-297.

Marty, Paul. 2017. Implicatures in the DP Domain: Massachusetts Institute of Technology dissertation.

Marty, Paul \& Jacopo Romoli. 2020. Presuppositions, implicatures, and contextual equivalence. Natural Language Semantics https://semanticsarchive.net/Archive/ Tg2NzkyM/contextual.pdf.

Percus, Orin. 2006. Antipresuppositions. In Aymui Ueyama (ed.), Theoretical and empirical studies of reference and anaphora: Toward the establishment of generative grammar as empirical science, Japan Society for the Promotion of Science.

Rees, Alice \& Lewis Bott. 2018. The role of alternative salience in the derivation of scalar implicatures. Cognition 176. 1-14.

Rouillard, Vincent \& Bernhard Schwarz. 2017. Epistemic narrowing for maximize presupposition. In Andrew Lamont \& Katerina A. Tetzloff (eds.), Proceedings of North East Linguistic Society 47, 49-62.

Sauerland, Uli. 2008. Implicated presuppositions. In Sentence and context, Mouton de Gruyter.

Schlenker, Philippe. 2012. Maximize Presupposition and Gricean Reasoning. Natural Language Semantics 20 (4). 391-429.

Schneider, Cosima, Nadine Bade, Michael Franke \& Markus Janczyk. 2020. Presuppositions of determiners are immediately used to disambiguate utterance meaning: A mouse-tracking study on the german language. Psychological Research 1-19.

Schneider, Cosima, Carolin Schonard, Michael Franke, Gerhard Jäger \& Markus Janczyk. 2019. Pragmatic processing: An investigation of the (anti-)presuppositions of determiners using mouse-tracking. Cognition 193. 104024. https://doi.org/10.1016/j.cognition.2019.104024.

Singh, Raj. 2011. Maximize Presupposition! and Local Contexts. Natural Language Semantics 19. 149-168.

Spector, Benjamin \& Yasutada Sudo. 2017. Presupposed ignorance and exhaustification: how scalar implicatures and presuppositions interact. Linguistics and Philosophy 40(5). 473-517. 10.1007/s10988-017-9208-9. 